\title{
Controlled Lagrangians and Stabilization of the Discrete Cart-Pendulum System
}

\author{
Anthony M. Bloch, Melvin Leok \\ Department of Mathematics \\ University of Michigan \\ Ann Arbor, MI 48109 \\ \{abloch, mleok\}@umich.edu
}

\author{
Jerrold E. Marsden \\ Control and Dynamical Systems \\ California Institute of Technology \\ Pasadena, CA 91125 \\ marsdenecds.caltech.edu
}

\author{
Dmitry V. Zenkov \\ Department of Mathematics \\ North Carolina State University \\ Raleigh, NC 27695 \\ dvzenkov@unity.ncsu.edu
}

\begin{abstract}
Matching techniques are developed for discrete mechanical systems with symmetry. We describe new phenomena that arise in the controlled Lagrangian approach for mechanical systems in the discrete context. In particular, one needs to either make an appropriate selection of momentum levels or introduce a new parameter into the controlled Lagrangian to complete the matching procedure. We also discuss digital and model predictive control.
\end{abstract}

\section{INTRODUCTION}

The method of controlled Lagrangians for stabilization of relative equilibria (steady state motions) originated in Bloch, Leonard, and Marsden [3] and was then developed in Auckly [1], Bloch, Leonard, and Marsden [4], [5], [6], Bloch, Chang, Leonard, and Marsden [7], and Hamberg [10], [11]. A similar approach for Hamiltonian controlled systems was introduced and further studied in the work of Blankenstein, Ortega, van der Schaft, Maschke, Spong, and their collaborators (see, e.g., [18] and related references). The two methods were shown to be equivalent in [8] and a nonholonomic version was developed in [20], [21], and [2].

According to the method of controlled Lagrangians, the original controlled system is represented as a new, uncontrolled Lagrangian system for a suitable controlled Lagrangian. The energy associated with this controlled Lagrangian is designed to be positive or negative definite at the (relative) equilibrium to be stabilized. The time-invariant feedback control law is obtained from the equivalence requirement for the new and old systems of equations of motion. If asymptotic stabilization is desired, dissipation-emulating terms are added to the control input.

In this paper the method of controlled Lagrangians is applied to the discrete cart-pendulum system. This study is motivated by the importance of structure-preserving algorithms for numerical simulation of controlled systems. In particular, as the closed loop dynamics of a controlled Lagrangian system is itself Lagrangian, it is natural to adopt a variational discretization that exhibits good long-time numerical stability.

We carry out the matching procedure explicitly for the discrete cart-pendulum system and prove that we can asymptotically stabilize the upward vertical position of the pendulum. The theoretical analysis is validated by simulating the discrete cart-pendulum system with control, and when dissipation is added, the inverted pendulum configuration is asymptotically stabilized, as predicted.

We then use the discrete controlled dynamics to construct a real-time model predictive controller with piecewise constant control inputs. This serves to illustrate how discrete mechanics can be naturally applied to yield digital controllers for mechanical systems.

The paper is organized as follows: In Sections II and III we review discrete mechanics and the method of controlled Lagrangians for stabilization of (relative) equilibria of mechanical systems. The discrete version of the method of controlled Lagrangians is discussed in Section IV. The theory is illustrated with the discrete cart-pendulum system in Section V. Simulations and the construction of the digital controller are presented in Sections VI and VII.

In a future publication we intend to treat discrete systems with nonabelian symmetries as well as systems with nonholonomic constraints.

\section{An Overview of Discrete Mechanics}

A discrete analogue of Lagrangian mechanics can be obtained by considering a discretization of Hamilton's principle; this approach underlies the construction of variational integrators. See Marsden and West [17] and references therein for a more detailed discussion of discrete mechanics.

A key notion is that of the discrete Lagrangian, which is a map $L^{d}: Q \times Q \rightarrow \mathbb{R}$ that approximates the action integral along an exact solution of the Euler-Lagrange equations joining $q_{k}$ and $q_{k+1}$,

$$
L^{d}\left(q_{k}, q_{k+1}\right) \approx \operatorname{ext}_{q \in \mathcal{C}([0, h], Q)} \int_{0}^{h} L(q, \dot{q}) d t
$$

where $\mathcal{C}([0, h], Q)$ is the space of curves $q:[0, h] \rightarrow Q$ with $q(0)=q_{k}, q(h)=q_{k+1}$ and ext denotes extremum.

In the discrete setting, the action integral of Lagrangian mechanics is replaced by an action sum

$$
S^{d}=\sum_{k=0}^{N-1} L^{d}\left(q_{k}, q_{k+1}\right),
$$

where $q_{k} \in Q$. The equations are obtained by the discrete Hamilton's principle which extremizes the discrete action given fixed endpoints $q_{0}$ and $q_{N}$. Taking the extremum over $q_{1}, \ldots, q_{N-1}$ gives the discrete Euler-Lagrange equations

$$
D_{1} L^{d}\left(q_{k}, q_{k+1}\right)+D_{2} L^{d}\left(q_{k-1}, q_{k}\right)=0,
$$


for $k=1, \ldots, N-1$. This implicitly defines the update map $\Phi: Q \times Q \rightarrow Q \times Q$, where $\Phi\left(q_{k-1}, q_{k}\right)=\left(q_{k}, q_{k+1}\right)$ and $Q \times Q$ replaces the phase space $T Q$ of Lagrangian mechanics. In the rest of this paper, we will adopt the notations

$$
q_{k+1 / 2}=\frac{q_{k}+q_{k+1}}{2}, \quad \Delta q_{k}=q_{k+1}-q_{k} .
$$

This allows us to express a second-order accurate discrete Lagrangian as

$$
L^{d}\left(q_{k}, k+1\right)=h L\left(q_{k+1 / 2}, \Delta q_{k} / h\right) .
$$

More generally, higher-order discrete Lagrangians can be obtained by using higher-order polynomial interpolation and numerical quadrature schemes. This yields the following approximation to (1):

$$
L^{d}\left(q_{k}, q_{k+1}\right)=\underset{q \in \mathcal{C}^{s}([0, h], Q)}{\operatorname{ext}} h \sum_{i=1}^{s} b_{i} L\left(q\left(c_{i} h\right), \dot{q}\left(c_{i} h\right)\right),
$$

where $c_{i}$ are a set of quadrature points, $b_{i}$ are the associated maximal order weights, and $\mathcal{C}^{s}([0, h], Q)=\{q \in$ $\mathcal{C}([0, h], Q) \mid q$ is a polynomial of degree $s\}$. The discrete Lagrangian (2) arises from this general formulation by using linear interpolation and the midpoint rule.

Since we are concerned with control, we need to consider the effect of external forces on Lagrangian systems. In the context of discrete mechanics, this is addressed by introducing the discrete Lagrange-d'Alembert principle (see, Kane, Marsden, Ortiz, West [14]), which states that

$$
\delta \sum_{k=0}^{n-1} L^{d}\left(q_{k}, q_{k+1}\right)+\sum_{k=0}^{n-1} F^{d}\left(q_{k}, q_{k+1}\right) \cdot\left(\delta q_{k}, \delta q_{k+1}\right)=0
$$

for all variations $\boldsymbol{\delta} \boldsymbol{q}$ of $\boldsymbol{q}$ that vanish at the endpoints. Here, $\boldsymbol{q}$ denotes the vector of positions $\left(q_{0}, q_{1}, \ldots, q_{N}\right)$, and $\boldsymbol{\delta} \boldsymbol{q}=\left(\delta q_{0}, \delta q_{1}, \ldots, \delta q_{N}\right)$, where $\delta q_{k} \in T_{q_{k}} \mathcal{C}(Q)$. The discrete one-form $F^{d}$ on $Q \times Q$ approximates the impulse integral between the points $q_{k}$ and $q_{k+1}$, just as the discrete Lagrangian $L^{d}$ approximates the action integral. We define the one-forms $F_{+}^{d}$ and $F_{-}^{d}$ on $Q \times Q$ and the maps $F_{1}^{d}, F_{2}^{d}$ : $Q \times Q \rightarrow T^{*} Q$ by the relations

$$
\begin{aligned}
F_{+}^{d}\left(q_{0}, q_{1}\right) \cdot\left(\delta q_{0}, \delta q_{1}\right) & =F_{2}^{d}\left(q_{0}, q_{1}\right) \cdot \delta q_{1} \\
& :=F^{d}\left(q_{0}, q_{1}\right) \cdot\left(0, \delta q_{1}\right), \\
F_{-}^{d}\left(q_{0}, q_{1}\right) \cdot\left(\delta q_{0}, \delta q_{1}\right) & =F_{1}^{d}\left(q_{0}, q_{1}\right) \cdot \delta q_{0} \\
& :=F^{d}\left(q_{0}, q_{1}\right) \cdot\left(\delta q_{0}, 0\right) .
\end{aligned}
$$

The discrete Lagrange-d'Alembert principle may then be rewritten as

$$
\begin{aligned}
& \delta \sum_{k=0}^{n-1} L^{d}\left(q_{k}, q_{k+1}\right) \\
& +\sum_{k=0}^{n-1}\left[F_{1}^{d}\left(q_{k}, q_{k+1}\right) \cdot \delta q_{k}+F_{2}^{d}\left(q_{k}, q_{k+1}\right) \cdot \delta q_{k+1}\right]=0
\end{aligned}
$$

for all variations $\boldsymbol{\delta} \boldsymbol{q}$ of $\boldsymbol{q}$ that vanish at the endpoints. This is equivalent to the forced discrete Euler-Lagrange equations

$$
\begin{aligned}
D_{1} L^{d}\left(q_{k}, q_{k+1}\right) & +D_{2} L^{d}\left(q_{k-1}, q_{k}\right) \\
& +F_{1}^{d}\left(q_{k}, q_{k+1}\right)+F_{2}^{d}\left(q_{k-1}, q_{k}\right)=0 .
\end{aligned}
$$

\section{Matching AND CONTROLled LAGRANGiAns}

In the controlled Lagrangian approach one considers a mechanical system with an uncontrolled (free) Lagrangian equal to kinetic energy minus potential energy. In the simplest setting we modify the kinetic energy to produce a new controlled Lagrangian which describes the dynamics of the controlled closed-loop system. The method may be extended in various ways including the incorporation of potential shaping.

Suppose our system has configuration space $Q$ and a Lie group $G$ acts freely and properly on $Q$. It is useful to keep in mind the case in which $Q=S \times G$ with $G$ acting only on the second factor by the left group multiplication.

For example, for the inverted planar pendulum on a cart, $Q=S^{1} \times \mathbb{R}$ with $G=\mathbb{R}$, the group of reals under addition (corresponding to translations of the cart).

Our goal is to control the variables lying in the shape space $Q / G$ using controls that act directly on the variables lying in $G .^{1}$ The controlled Lagrangian is constructed to be $G$ invariant, thus providing modified or controlled conservation laws. In this paper we assume that $G$ is an abelian group.

The key modification of the Lagrangian involves changing the kinetic energy metric $g(\cdot, \cdot)$. The tangent space to $Q$ can be split into a sum of horizontal and vertical parts defined as follows: For each tangent vector $v_{q}$ to $Q$ at a point $q \in Q$, we can write a unique decomposition $v_{q}=\operatorname{Hor} v_{q}+\operatorname{Ver} v_{q}$, such that the vertical part is tangent to the orbits of the $G$-action and the horizontal part is metric-orthogonal to the vertical space, i.e., it is uniquely defined by the identity

$$
g\left(v_{q}, w_{q}\right)=g\left(\operatorname{Hor} v_{q}, \operatorname{Hor} w_{q}\right)+g\left(\operatorname{Ver} v_{q}, \operatorname{Ver} w_{q}\right)
$$

with $v_{q}$ and $w_{q}$ arbitrary tangent vectors to $Q$ at the point $q \in Q$. This choice of horizontal space coincides with that given by the mechanical connection; see, for example, Marsden [1992].

For the kinetic energy of our controlled Lagrangian, we use a modified version of the right-hand side of equation (4). The potential energy remains unchanged. The modification consists of three ingredients:

1) a new choice of horizontal space, denoted $\mathrm{Hor}_{\tau}$,

2) a change $g \rightarrow g_{\sigma}$ of the metric on horizontal vectors,

3) a change $g \rightarrow g_{\rho}$ of the metric on vertical vectors.

Let $\xi_{Q}$ denote the infinitesimal generator corresponding to $\xi \in \mathfrak{g}$, where $\mathfrak{g}$ is the Lie algebra of $G$ (see Marsden [1992] or Marsden and Ratiu [1994]). Thus, for each $\xi \in \mathfrak{g}, \xi_{Q}$ is a vector field on the configuration manifold $Q$ and its value at a point $q \in Q$ is denoted $\xi_{Q}(q)$.

Definition 1: Let $\tau$ be a Lie-algebra-valued horizontal one-form on $Q$; that is, a one-form that annihilates vertical vectors. The $\tau$-horizontal space at $q \in Q$ consists of tangent vectors to $Q$ at $q$ of the form $\operatorname{Hor}_{\tau} v_{q}=\operatorname{Hor} v_{q}-[\tau(v)]_{Q}(q)$, which also defines $v_{q} \mapsto \operatorname{Hor}_{\tau}\left(v_{q}\right)$, the $\tau$-horizontal projection. The $\tau$-vertical projection operator is defined by $\operatorname{Ver}_{\tau}\left(v_{q}\right):=\operatorname{Ver}\left(v_{q}\right)+[\tau(v)]_{Q}(q)$.

\footnotetext{
${ }^{1}$ The shape space is $S$ in the case $Q=S \times G$.
} 
Definition 2: Given $g_{\sigma}, g_{\rho}$ and $\tau$, the controlled Lagrangian equals a modified kinetic minus the given potential energy:

$$
\begin{aligned}
L_{\tau, \sigma, \rho}(v)=\frac{1}{2}\left[g_{\sigma}\left(\operatorname{Hor}_{\tau} v_{q}, \operatorname{Hor}_{\tau} v_{q}\right)\right. & \\
& \left.+g_{\rho}\left(\operatorname{Ver}_{\tau} v_{q}, \operatorname{Ver}_{\tau} v_{q}\right)\right]-V(q) .
\end{aligned}
$$

The equations corresponding to this Lagrangian will be our closed-loop equations. The new terms appearing in those equations corresponding to the directly controlled variables are interpreted as control inputs. The modifications to the Lagrangian are chosen so that no new terms appear in the equations corresponding to the variables that are not directly controlled. We refer to this process as matching.

Once the control law is derived using the controlled Lagrangian, the closed-loop stability of an equilibrium can be determined by energy methods, using any available freedom in the choice of $\tau, g_{\sigma}$ and $g_{\rho}$.

Under some reasonable assumptions on the metric $g_{\sigma}$, $L_{\tau, \sigma, \rho}(v)$ has the following useful structure.

Theorem 3: Assume that $g=g_{\sigma}$ on Hor and Hor and Ver are orthogonal for $g_{\sigma}$. Then

$L_{\tau, \sigma, \rho}(v)=L\left(v+\tau(v)_{Q}\right)+\frac{1}{2} g_{\sigma}\left(\tau(v)_{Q}, \tau(v)_{Q}\right)+\frac{1}{2} \varpi(v)$, where $v \in T_{q} Q$ and $\varpi(v)=\left(g_{\rho}-g\right)\left(\operatorname{Ver}_{\tau}(v), \operatorname{Ver}_{\tau}(v)\right)$.

A useful example treated in earlier papers in the smooth setting is the pendulum on a cart. Let $s$ denote the position of the cart on the $s$-axis and let $\theta$ denote the angle of the pendulum with the upright vertical, as in Figure 1. The

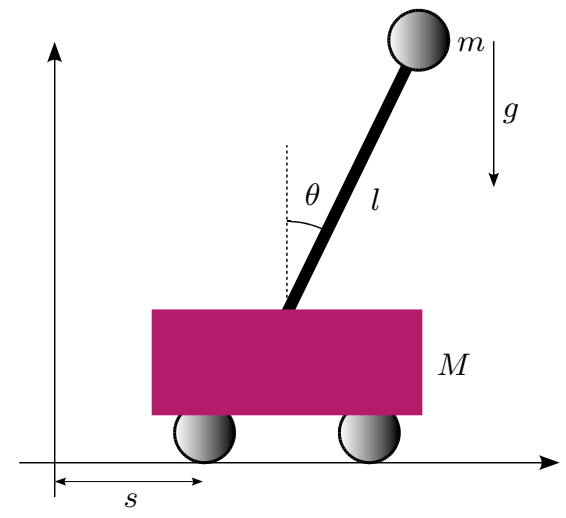

Fig. 1. The pendulum on a cart

configuration space for this system is $Q=S \times G=S^{1} \times \mathbb{R}$, with the first factor being the pendulum angle $\theta$ and the second factor being the cart position $s$. The velocity phase space, $T Q$, has coordinates $(\theta, s, \dot{\theta}, \dot{s})$. The length of the pendulum is $l$, the mass of the pendulum is $m$ and that of the cart is $M$.

The symmetry group $G$ of the pendulum-cart system is that of translation in the $s$ variable, so $G=\mathbb{R}$. We do not destroy this symmetry when doing stabilization in $\theta$.

For notational convenience, write the Lagrangian as

$$
L(\theta, s, \dot{\theta}, \dot{s})=\frac{1}{2}\left(\alpha \dot{\theta}^{2}+2 \beta \cos \theta \dot{s} \dot{\theta}+\gamma \dot{s}^{2}\right)-U(\theta),
$$

where $\alpha=m l^{2}, \beta=m l, \gamma=M+m$ are constants and $U(\theta)=-m g l \cos \theta$ is the potential energy. Note that $\alpha \gamma-$ $\beta^{2}>0$.

\section{Discrete MatChing}

In discretizing the method of controlled Lagrangians, it is natural to combine the results of Theorem 3 with formula (3). To simplify the exposition in the remainder of the paper, we will restrict ourselves to the second-order controlled discrete Lagrangian that is defined by

$$
\begin{aligned}
& L_{\tau, \sigma, \rho}^{d}\left(q_{k}, q_{k+1}\right)=h\left[L\left(q_{k+\frac{1}{2}}, \Delta q_{k} / h+\tau\left(\Delta q_{k} / h\right)_{Q}\right)\right. \\
& \left.\quad+\frac{1}{2} g_{\sigma}\left(\tau\left(\Delta q_{k} / h\right)_{Q}, \tau\left(\Delta q_{k} / h\right)_{Q}\right)+\frac{1}{2} \varpi\left(\Delta q_{k} / h\right)\right] .
\end{aligned}
$$

An alternative approach for parameterizing the space of controlled Lagrangians is through the use of discrete connections (see Leok, Marsden, and Weinstein [15]).

\section{Stabilization of the Discrete Cart-Pendulum SYSTEM}

\section{A. The Discrete Cart-Pendulum}

For the discrete cart-pendulum system, $q_{k}=\left(\theta_{k}, s_{k}\right)$. According to (2), the discrete Lagrangian for the cartpendulum system is

$$
L^{d}\left(q_{k}, q_{k+1}\right)=h L\left(\theta_{k+\frac{1}{2}}, \Delta \theta_{k} / h, \Delta s_{k} / h\right),
$$

where $L$ is Lagrangian (5). The discrete dynamics is governed by the equations

$$
\begin{aligned}
& \frac{\partial L^{d}\left(q_{k}, q_{k+1}\right)}{\partial \theta_{k}}+\frac{\partial L^{d}\left(q_{k-1}, q_{k}\right)}{\partial \theta_{k}}=0, \\
& \frac{\partial L^{d}\left(q_{k}, q_{k+1}\right)}{\partial s_{k}}+\frac{\partial L^{d}\left(q_{k-1}, q_{k}\right)}{\partial s_{k}}=u_{k},
\end{aligned}
$$

where $u_{k}$ is the control input. Straightforward calculation shows that the relative equilibria $\theta_{k}=0, s_{k}=$ const of the discrete cart-pendulum are unstable.

\section{B. Discrete Matching Conditions}

Using (6), we define the discrete controlled Lagrangian $L_{\tau, \sigma}^{d}\left(q_{k}, q_{k+1}\right)$ for the cart-pendulum system by

$$
\begin{aligned}
h L\left(\theta_{k+\frac{1}{2}}, \Delta \theta_{k} / h, \Delta s_{k} / h\right. & \left.+\tau\left(\theta_{k+\frac{1}{2}}\right) \Delta \theta_{k} / h\right) \\
& +\frac{h}{2} \sigma \gamma\left(\tau\left(\theta_{k+\frac{1}{2}}\right) \Delta \theta_{k} / h\right)^{2},
\end{aligned}
$$

where $L$ is Lagrangian of the continuous-time cart-pendulum system (5).

The dynamics associated with (9) is

$$
\begin{aligned}
& \frac{\partial L_{\tau, \sigma}^{d}\left(q_{k}, q_{k+1}\right)}{\partial \theta_{k}}+\frac{\partial L_{\tau, \sigma}^{d}\left(q_{k-1}, q_{k}\right)}{\partial \theta_{k}}=0, \\
& \frac{\partial L_{\tau, \sigma}^{d}\left(q_{k}, q_{k+1}\right)}{\partial s_{k}}+\frac{\partial L_{\tau, \sigma}^{d}\left(q_{k-1}, q_{k}\right)}{\partial s_{k}}=0 .
\end{aligned}
$$

Equation (11) is equivalent to the discrete controlled momentum conservation:

$$
p_{k}=\mu,
$$


where

$$
p_{k}=-\frac{\beta \Delta \theta_{k} \cos \theta_{k+1 / 2}+\gamma \Delta s_{k}+\gamma \Delta \theta_{k} \tau\left(\theta_{k+1 / 2}\right)}{h} .
$$

Setting

$$
u_{k}=\frac{\gamma \Delta \theta_{k} \tau\left(\theta_{k+1 / 2}\right)-\gamma \Delta \theta_{k-1} \tau\left(\theta_{k-1 / 2}\right)}{h}
$$

matches equations (8) and (11) and allows one to represent the discrete momentum equation (8) as the discrete momentum conservation law

$$
p_{k}=p \text {. }
$$

Define the functions $F(\theta)$ and $G_{\mu}(\theta)$ by the formulae $F(\theta)=\beta \gamma \cos \theta+\gamma^{2} \sigma \tau(\theta)$ and $G_{\mu}(\theta)=h^{2} \beta(\mu-p) \cos \theta+$ $h^{2} \gamma \mu \tau(\theta)$.

Theorem 4: The dynamics (7) and (8) restricted to the momentum level $p_{k}=p$ is equivalent to the dynamics (10) and (11) restricted to the momentum level $p_{k}=\mu$ if and only if the matching condition

$$
\begin{gathered}
{\left[\Delta \theta_{k-1}^{2} \tau\left(\theta_{k-\frac{1}{2}}\right) F^{\prime}\left(\theta_{k-\frac{1}{2}}\right)-\Delta \theta_{k}^{2} \tau\left(\theta_{k+\frac{1}{2}}\right) F^{\prime}\left(\theta_{k+\frac{1}{2}}\right)\right]} \\
+2\left[\Delta \theta_{k-1} \tau\left(\theta_{k-\frac{1}{2}}\right) F\left(\theta_{k-\frac{1}{2}}\right)+\Delta \theta_{k} \tau\left(\theta_{k+\frac{1}{2}}\right) F\left(\theta_{k+\frac{1}{2}}\right)\right] \\
+\left[\Delta \theta_{k-1} G_{\mu}^{\prime}\left(\theta_{k-\frac{1}{2}}\right)+\Delta \theta_{k} G_{\mu}^{\prime}\left(\theta_{k+\frac{1}{2}}\right)\right] \\
+2\left[G_{\mu}\left(\theta_{k-\frac{1}{2}}\right)-G_{\mu}\left(\theta_{k+\frac{1}{2}}\right)\right]=0
\end{gathered}
$$

holds.

Proof: Solve equations (12) and (15) for $\Delta s_{k}$ and substitute the solutions in equations (7) and (10), respectively. This process is a simple version of discrete reduction [12]. A computation shows that the equations obtained this way are equivalent if and only if (16) is satisfied.

Corollary 5: The matching condition (16) is satisfied if

$$
\tau(\theta)=\kappa \cos \theta, \quad \sigma=-\frac{\beta}{\gamma \kappa}, \quad \mu=\frac{\beta}{\beta+\gamma \kappa} p .
$$

Proof: Equation (17) implies $F(\theta)=G(\theta)=0$, and therefore each term in the left-hand side of equation (16) vanishes. Note that the momentum levels $p$ and $\mu$ are not the same.

We now briefly discuss an alternative matching procedure. Define the discrete controlled Lagrangian $\Lambda_{\tau, \sigma, \lambda}^{d}\left(q_{k}, q_{k+1}\right)$ by the formula

$$
\begin{aligned}
& h L\left(\theta_{k+\frac{1}{2}}, \Delta \theta_{k} / h, \Delta s_{k} / h+\tau\left(\theta_{k+\frac{1}{2}}\right) \Delta \theta_{k} / h\right) \\
& \quad+\frac{h}{2} \sigma \gamma\left(\tau\left(\theta_{k+\frac{1}{2}}\right) \Delta \theta_{k} / h\right)^{2}+h \lambda \tau\left(\theta_{k+\frac{1}{2}}\right) \Delta \theta_{k} .
\end{aligned}
$$

The dynamics associated with this Lagrangian is

$$
\begin{gathered}
\frac{\partial \Lambda_{\tau, \sigma, \lambda}^{d}\left(q_{k}, q_{k+1}\right)}{\partial \theta_{k}}+\frac{\partial \Lambda_{\tau, \sigma, \lambda}^{d}\left(q_{k-1}, q_{k}\right)}{\partial \theta_{k}}=0, \\
\frac{\partial \Lambda_{\tau, \sigma}^{d}\left(q_{k}, q_{k+1}\right)}{\partial s_{k}}+\frac{\partial \Lambda_{\tau, \sigma}^{d}\left(q_{k-1}, q_{k}\right)}{\partial s_{k}}=0 .
\end{gathered}
$$

As before, the discrete controlled momentum is given by formula (13), and equation (19) is equivalent to the discrete momentum conservation (15).
Theorem 6: The dynamics (7) and (8) restricted to the momentum level $p_{k}=p$ is equivalent to the dynamics (18) and (19) restricted to the same momentum level if and only if the matching condition

$$
\begin{aligned}
& {\left[\Delta \theta_{k-1}^{2} \tau\left(\theta_{k-\frac{1}{2}}\right) F^{\prime}\left(\theta_{k-\frac{1}{2}}\right)-\Delta \theta_{k}^{2} \tau\left(\theta_{k+\frac{1}{2}}\right) F^{\prime}\left(\theta_{k+\frac{1}{2}}\right)\right]} \\
& +2\left[\Delta \theta_{k-1} \tau\left(\theta_{k-\frac{1}{2}}\right) F\left(\theta_{k-\frac{1}{2}}\right)+\Delta \theta_{k} \tau\left(\theta_{k+\frac{1}{2}}\right) F\left(\theta_{k+\frac{1}{2}}\right)\right] \\
& +h^{2} \gamma(p+\lambda)\left[2 \tau\left(\theta_{k-\frac{1}{2}}\right)-2 \tau\left(\theta_{k+\frac{1}{2}}\right)\right. \\
& \left.\quad+\Delta \theta_{k-1} \tau^{\prime}\left(\theta_{k-\frac{1}{2}}\right)+\Delta \theta_{k} \tau^{\prime}\left(\theta_{k+\frac{1}{2}}\right)\right]=0
\end{aligned}
$$

holds.

Note that in this case we add an extra term to the controlled Lagrangian which eliminates the need for adjusting the momentum level. The proof of Theorem 6 is similar to that of Theorem 4. Further details will be given in a forthcoming publication.

Corollary 7: The matching condition (20) is satisfied for the cart-pendulum system if

$$
\tau(\theta)=\kappa \cos \theta, \quad \sigma=-\frac{\beta}{\gamma \kappa}, \quad \lambda=-p .
$$

Remark. The quantities $\tau(\theta)$ and $\sigma$ obtained in Corollaries 5 and 7 are identical to those resulting from the continuous-time matching procedure for the cart-pendulum system.

\section{Stabilization of the Discrete Cart-Pendulum System}

Here we study the stability properties of the relative equilibria $\theta_{k}=0, s_{k}=$ const of the discrete cart-pendulum system.

Theorem 8: The relative equilibria $\theta_{k}=0, s_{k}=$ const of equations (7) and (8), with $u_{k}$ defined by (14), are spectrally stable if

$$
\kappa>\frac{\alpha \gamma-\beta^{2}}{\beta \gamma} .
$$

Proof: Recall that $U^{\prime \prime}(0)=-C$, where $C>0$. The linearization of the reduced dynamics (7) and (8) at $\theta=0$ is computed to be

$$
\begin{aligned}
\frac{\alpha \gamma-\beta^{2}-\beta \gamma \kappa}{h^{2} \gamma}( & \left.\Delta \theta_{k-1}-\Delta \theta_{k}\right) \\
+ & \frac{C}{4}\left(\theta_{k-1}+2 \theta_{k}+\theta_{k+1}\right)=0 .
\end{aligned}
$$

Observe that the value of $p$ does not affect the linearized dynamics.

The linearized dynamics preserves the quadratic approximation of the discrete energy

$$
\frac{\alpha \gamma-\beta^{2}-\beta \gamma \kappa}{2 h^{2} \gamma} \Delta \theta_{k}^{2}-\frac{C \theta_{k+1 / 2}^{2}}{2} .
$$

The equilibrium $\theta_{k}=0$ of (22) is stable if and only if the function (23) is negative-definite at $\theta_{k}=\theta_{k+1}=0$. The latter requirement is equivalent to condition (21).

Remark. Stability condition (21) is identical to the stability condition of the continuous-time cart-pendulum system. 
The spectrum of the linear map $\left(\theta_{k-1}, \theta_{k}\right) \mapsto\left(\theta_{k}, \theta_{k+1}\right)$ defined by (22) belongs to the unit circle. Spectral stability in this situation is not sufficient to conclude nonlinear stability.

We now modify the control input (14) by adding the discrete dissipation-emulating term

$$
-\frac{D\left(\Delta \theta_{k-1}+\Delta \theta_{k}\right)}{h}
$$

in order to achieve the asymptotic stabilization of the upward position of the pendulum. In the above, $D$ is a positive constant. The discrete momentum conservation law becomes

$$
p_{k}=p-\frac{D \theta_{k+1 / 2}}{h} .
$$

Straightforward calculation shows that the spectrum of the matrix of the linear map $\left(\theta_{k-1}, \theta_{k}\right) \mapsto\left(\theta_{k}, \theta_{k+1}\right)$ defined by the reduced discrete dynamics belongs to the open unit disc. This implies that the equilibrium $\theta=0$ is asymptotically stable.

\section{Simulations}

Simulating the discrete behavior of the controlled Lagrangian system is simply a matter of viewing the forced discrete Euler-Lagrange equation as an implicit update map $\Phi:\left(q_{k-2}, q_{k-1}\right) \mapsto\left(q_{k-1}, q_{k}\right)$. This presupposes that the initial conditions are given in the form $\left(q_{0}, q_{1}\right)$, however it might be preferable to specify the initial conditions as $\left(q_{0}, \dot{q}_{0}\right)$ instead. In this situation, one solves the boundary condition

$$
\frac{\partial L}{\partial \dot{q}}\left(q_{0}, \dot{q}_{0}\right)+D_{1} L^{d}\left(q_{0}, q_{1}\right)+F_{1}^{d}\left(q_{0}, q_{1}\right)=0
$$

for $q_{1}$, which can then be time marched using the implicit update map $\Phi$. This procedure is described in Algorithm 1.

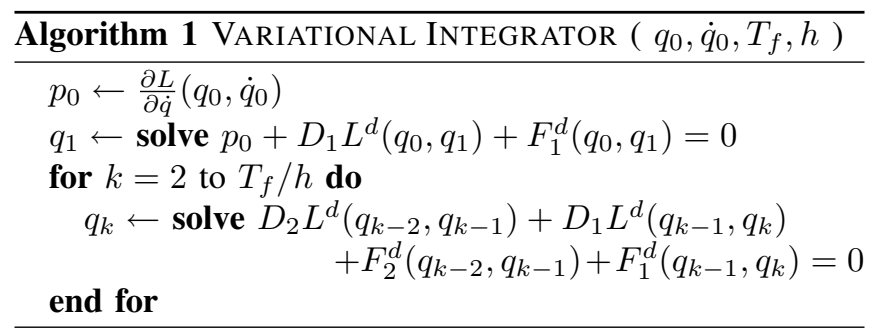

When $\kappa$ is twice the critical value, and dissipation is added, the $\theta$ dynamics is asymptotically stabilized, as predicted. This is illustrated in Figure 2.

\section{ViI. Model Predictive Controller}

We now explore the use of the forced discrete EulerLagrange equations as a model for use in the context of a real-time model predictive controller, with piecewise constant control forces. Algorithm 2 describes the procedure in detail. The digital controller uses the position information it senses for $t=-2 h,-h$ to estimate the positions at $t=0, h, 2 h$ during the time interval $t=[-h, 0]$. This allows
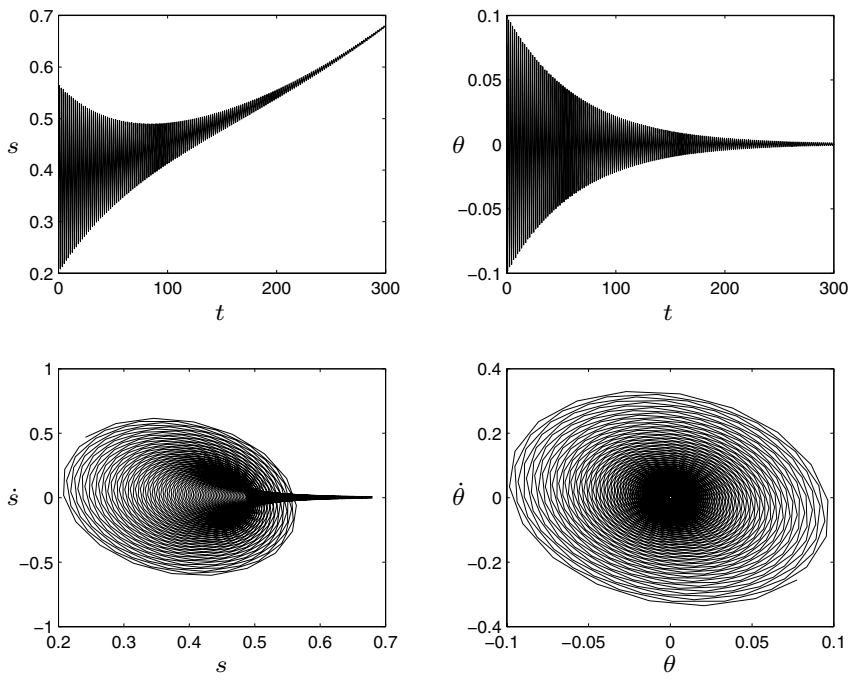

Fig. 2. Controlled dynamics with dissipation

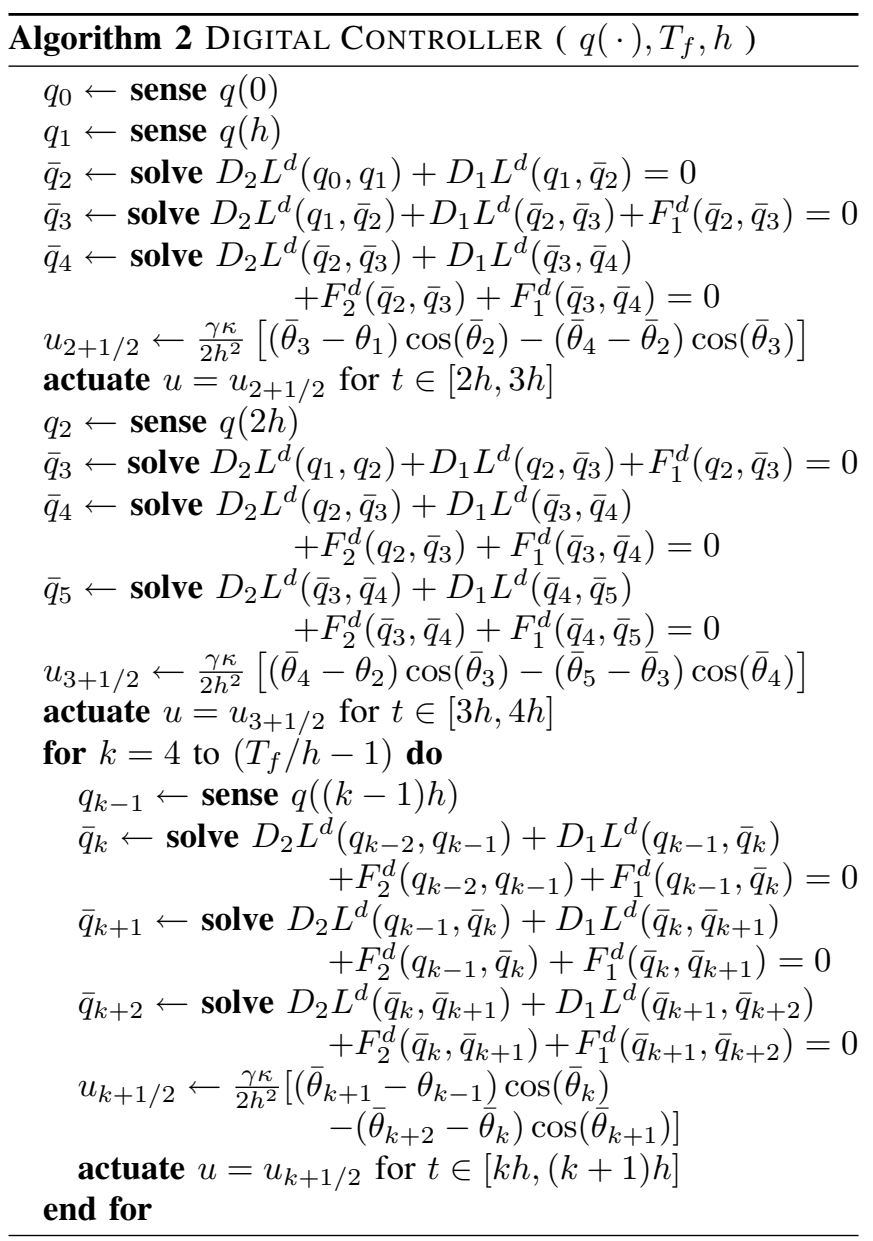


it to compute a symmetric finite difference approximation to the control force at $t=h / 2$ using the approximation

$$
\begin{aligned}
u(h / 2) & =-\left.\frac{d}{d t} \gamma \tau(\theta) \dot{\theta}\right|_{t=h / 2} \\
& \approx \frac{\gamma \kappa}{2 h^{2}}\left[\left(\bar{\theta}_{1}-\theta_{-1}\right) \cos \left(\bar{\theta}_{0}\right)-\left(\bar{\theta}_{2}-\bar{\theta}_{0}\right) \cos \left(\bar{\theta}_{1}\right)\right]
\end{aligned}
$$

where the overbar indicates that the position variable is being estimated by the numerical model. This control is then applied as a constant control input for the time interval $[0, h]$. If the three forward solves can be computed within the time interval $h$, then this algorithm can be implemented in realtime.

The initialization of the discrete controller is somewhat involved, since the system is unforced during the time interval $[0,2 h]$ while the controller senses the initial states, and computes the appropriate control forces. As a consequence, we initially have to solve a combination of the discrete EulerLagrange equations and the forced discrete Euler-Lagrange equations to estimate the evolution of the system, until the feedback actuation comes fully online.

The numerical simulation of the digital controller is shown in Figure 3. We see that the system is asymptotically stabilized in the $\theta$ variable. The use of a piecewise constant control introduces dissipation-like effects, which are reduced as the time-step is decreased. The nature of this behavior will be studied in a forthcoming publication.
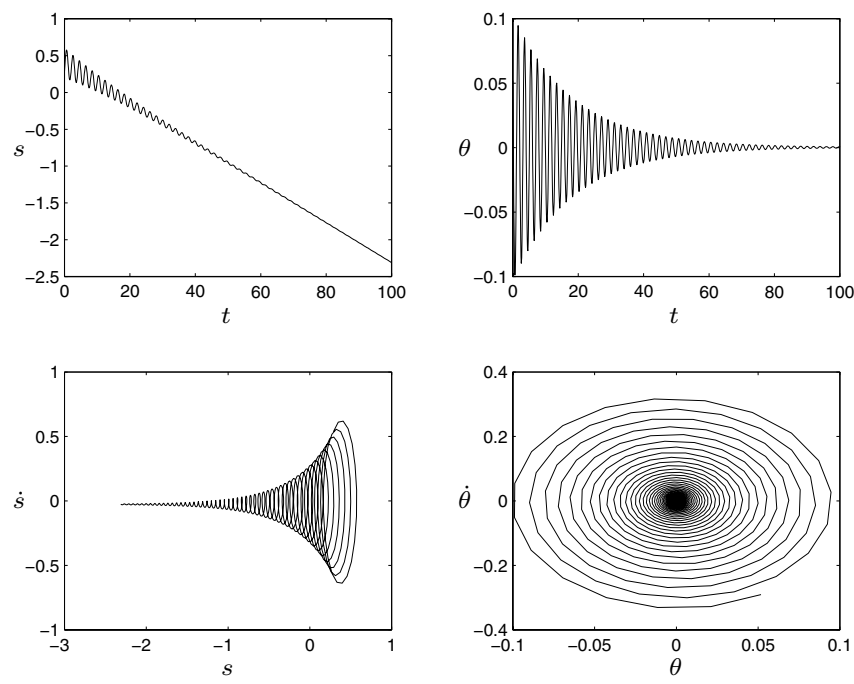

Fig. 3. Real-time piecewise constant model predictive controller

\section{CONCLUSIONS}

In this paper we have introduced the method of controlled Lagrangians for discrete systems and have shown that it leads to an effective numerical implementation for stabilization in the case of the discrete cart-pendulum model. The method in this paper is related to other discrete methods in control that have a long history; recent papers that use discrete mechanics in the context of optimal control and celestial navigation are
[9], [13], and [19]. The full theory of discrete controlled Lagrangians will be developed in a forthcoming paper.

\section{ACKNOWLEDGMents}

The authors would like to thank the reviewers for helpful remarks. The research of AMB was supported by NSF grants DMS-0103895, DMS-030583, and CMS-0408542. The research of ML was partially supported by NSF grant DMS0504747 and a University of Michigan Rackham faculty grant. The research of JEM was partially supported by ONR MURI Contract 00000916. The research of DVZ was partially supported by NSF grant DMS-0306017. AMB and DVZ would also like to thank the CRM, Barcelona, where part of this research was carried out.

\section{REFERENCES}

[1] Auckly, D., L. Kapitanski, \& W. White, Control of Nonlinear Underactuated Systems, Commun. Pure Appl. Math. 53, 2000, 354-369.

[2] Bloch, A. M., Nonholonomic Mechanics and Control, Interdisciplinary Appl. Math. 24, Springer-Verlag, 2003.

[3] Bloch, A. M., N. Leonard, \& J.E. Marsden, Stabilization of Mechanical Systems Using Controlled Lagrangians, Proc. CDC 36, 1997, 2356-2361.

[4] Bloch, A. M., N. Leonard, \& J. E. Marsden, Matching and Stabilization by the Method of Controlled Lagrangians, Proc. CDC 37, 1998, 14461451.

[5] Bloch, A. M., N. Leonard, \& J. E. Marsden, Potential Shaping and the Method of Controlled Lagrangians, Proc. CDC 38, 1999, 1652-1657.

[6] Bloch, A. M., N. E. Leonard, \& J. E. Marsden, Controlled Lagrangians and the Stabilization of Mechanical Systems I: The First Matching Theorem, IEEE Trans. on Systems and Control 45, 2000, 2253-2270.

[7] Bloch, A. M., D-E. Chang, N. E. Leonard, \& J. E. Marsden, Controlled Lagrangians and the Stabilization of Mechanical Systems II: Potential Shaping, Trans. IEEE on Autom. Contr. 46, 2001, 1556-1571.

[8] Chang, D-E., A. M. Bloch, N.E. Leonard, J.E. Marsden, \& C. Woolsey, The Equivalence of Controlled Lagrangian and Controlled Hamiltonian Systems, Control and the Calculus of Variations (special issue dedicated to J. L. Lions) 8, 2002, 393-422.

[9] Guibout, V. \& A. M. Bloch, A Discrete Maximum Principle for Solving Optimal Control Problems, Proc. CDC 43, 2004, 1806-1811.

[10] Hamberg, J., General Matching Conditions in the Theory of Controlled Lagrangians, Proc. CDC 38, 1999, 2519-2523.

[11] Hamberg, J., Controlled Lagrangians, Symmetries and Conditions for Strong Matching, In: Lagrangian and Hamiltonian Methods for Nonlinear Control, Elsevier, 2000.

[12] Jalnapurkar, S. M., M. Leok, J.E. Marsden \& M. West, Discrete Routh Reduction, 2005, arXiv:math.NA/0508330.

[13] Junge, O., J. Marsden, \& S. Ober-Blbaum, Discrete Mechanics and Optimal Control, Proc. of the 16th IFAC World Congress, 2005 (to appear).

[14] Kane, C., J.E. Marsden, M. Ortiz, \& M. West, Variational Integrators and the Newmark Algorithm for Conservative and Dissipative Mechanical Systems, Int. J. Numer. Math. Eng. 49, 2000, 1295-1325.

[15] Leok, M., J.E. Marsden, \& A.D. Weinstein, A Discrete Theory of Connections on Principal Bundles, 2005, arXiv:math.DG/0508338.

[16] Marsden, J. E., Lectures on Mechanics, London Mathematical Society Lecture Note Series 174, Cambridge University Press, 1992.

[17] Marsden, J.E. \& M. West, Discrete Mechanics and Variational Integrators, Acta Numerica 10, 2001, 357-514.

[18] Maschke, B., R. Ortega, \& A. van der Schaft, Energy-Based Lyapunov Functions for Forced Hamiltonian Systems with Dissipation, IEEE Trans. Automat. Control 45, 2001, 1498-1502.

[19] Sanyal, A., J. Shen, N. H. McClamroch, \& A. M. Bloch, Stability and Stabilization of Relative Equilibria of the Dumbbell Satellite in Central Gravity, 2005, Journal of the American Institute of Aeronautics and Astronautics, (to appear).

[20] Zenkov, D. V., A. M. Bloch, N. E. Leonard, \& J.E. Marsden, Matching and Stabilization of Low-Dimensional Nonholonomic Systems, Proc. CDC 39, 2000, 1289-1295.

[21] Zenkov, D. V., A. M. Bloch, \& J.E. Marsden, Flat Nonholonomic Matching, Proc. ACC, 2002, 2812-2817. 